\title{
Application of ArcGIS In the Evaluation of Land Intensive Use
}

\author{
Guang Yang, Xiaoshuo Bi, Zhihang Yao \\ College of Surveying and Geo-informatics \\ Tongji University \\ 1239Siping road, Shanghai, 200092, China \\ yangguang@tongji.edu.cn
}

\begin{abstract}
Land intensive use is essentially the relationship between land input and output, which means to get the highest output with the least input. The evaluation of land intensive use level is a vital content of urban land management, which can help to provide scientific basis for the governments' decision-making of land management. As a key tool of spatial analysis, ArcGIS plays a very important role in the evaluation of land intensive use. On the basis of discussing the importance of applying GIS to the evaluation work of land intensive use, this paper taking the work flow of the evaluation of intensive land use as the order to introduce the application of ArcGIS in the evaluation work, including image matching, data editing and production of thematic maps, etc., and puts forward the idea of optimizing this work in the future in order to provide reference for theapproaching evaluation of land intensive use and the application of ArcGIS software.
\end{abstract}

Keywords-Land evaluation; ArcGIS;Geodataset; Element class; Featureclass

\section{FOREWORD}

It is a great important strategic period now that in China, in which we have both opportunity and challenge in social development, and the pressure of land use will be strengthened. A intensive and efficient land use model in the development zone must be established to ensure a sustainable development of society and economy and to ease the contradiction between land supply and demand. The land intensive utilization evaluation work for the development zone can efficiently control blind expansion, help to tapping the potentiation of inefficiently-use land, enhance the ability of land to participate in macroeconomic regulation and control, and have great significance in building a resource-saving society .

In the past, operatorsis usually conduct data statistics and graph calculation and drawing work usually in the Office software and the AutoCAD platform. This kind of work mode has the advantage of simple operation, wide range of application,but the disadvantages are also obvious. Two separate work platform to the combination of graphic and attribute is not close, which often leads toa result of a difference between the final statistical results and the actual measured data.This will load heavy work to check. But with geographical information system (GIS) as a representative of modern science and technology, which can well achieve information acquisition, data processing, space application of automation, intellectualization and visualization,this kind of problem can be easily solved.

\section{APPLICATION OF GIS IN THE EVALUATION OF LAND INTENSIVE USE}

ArcGIS software is now one of the mostmainstream software in the area of geographic information engineering.It is a full range of the highest level of GIS products successfully launched by ESRI Company after the company integrated GIS with database, software engineering, artificial intelligence, network technology and othermainstream computer technology.In the process of land intensive use evaluation,generally the planning departments will provide the data with CAD format. ArcGIS can carry out the conversion withdiffidentformats of data, for example, data with CAD format can be converted to GIS format. What's more, the data can be edited, spatial query, attribute query, topology check and classification statistics and thematic map display using ArcGIS.The "model builder" in ArcGIS help to build a model easily, which can connect with a series of geographic processing tools to create a workflow.In addition,ArcGIS can also write the script program to expand thefunction, batch processing data, simplify the work flow and improve work efficiencyaccording to the special needs.It can provide convenient support to edit data and display high quality results to apply ArcGIS in the process of land intensive use evaluation.

\subsection{Image matching}

During the early period of evaluation, the research of the land nature and company information is needed. The remote sensing images can display the type of each block of land. Different types of land have obvious differences in remote sensing images and can be easily distinguished.Taking the image as anauxiliary basis for judge when carrying out the research can avoid or reduce the occurrence of injustice and ensure the result more accurate.

In the process of land intensive use evaluationland management department provides the remote sensing images and industrial block pictures in DWG format of the researched region. To overlay the remote sensing images and industrial block pictures will help users get auxiliary information for the research work. To realize the above effect, users can use image registration tool in ArcGIS software (called "geo-referencing") to establish and correct connection lines between the control points. At last, The superimposed picture can be derived and printed to be thereference maps for thefurther research. The effect of the superimposed picture is shown in "Fig.1": 


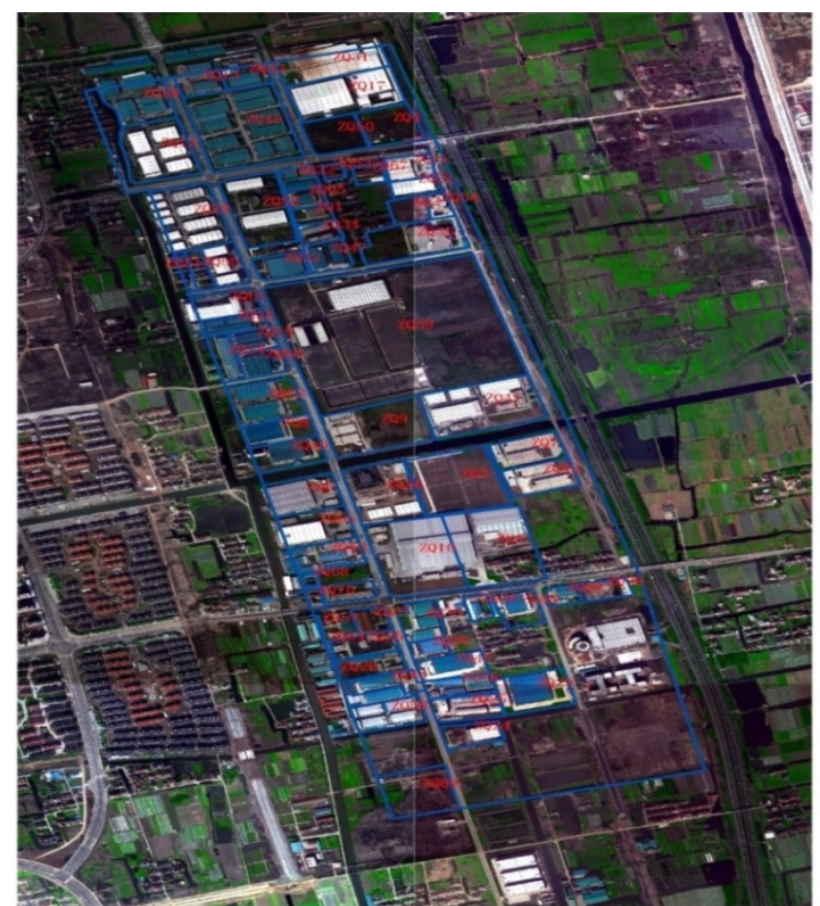

Figure 1. Overlaiad picture between picture of DWG format need toconstruct the planning CAD files provided by the land management department to construct the polygon.However, the plot plan is changing every year because when carrying out the land planning,the government will make somenew targets to the land. New and old planning maps tend to overlap. In addition, due to the CAD drawing is not standardized, between the adjacent plots, there may be some overlap or gaps, which may make no difference in the planning exhibition. But where import into ArcMap, after converted into polygon of ArcGIS format ,he pattern may have obvious defects somewhere,for example,there may be a blank region between patterns or one region may belongs to two or more patterns, which are not practical in the real world. Userscan useArcGIS's graphics editing function to correct the problemin order to eliminate these unreasonable phenomena.

The basic work is to find the wrong map spot .there are too many map spots when import from CAD to ArcGIS,so it will cost too much ifsimply check artificially. The topology checking tool inArcGIS can check the topological errorsautomatically,such as the coincidence of the line, intersection, unexpected and polygon overlap and so on. By selecting different validation rules, such as:"Must have no gaps”, "Must have no overlaps", users can check some particular situation and indicate the plots which have topological errors, as shown in "Fig.2".

\subsection{Data editing in ArcGIS}

\subsubsection{Editing graphicdata}

In the process of land intensive use evaluationusers

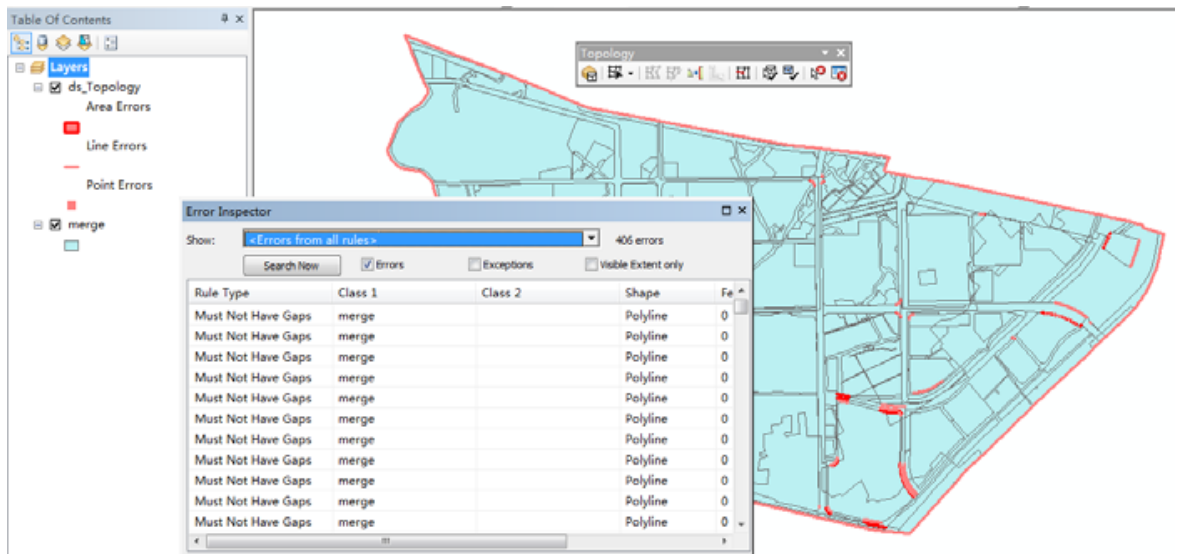

Figure 2. find the detect block by using the topological checker

It is necessary to edit and modify the map afterchecking out the error figure by using ArcGIS.The topological checking tool can be used to Batch modify and make new land ports of the Overlapping or gap-existing maps to eliminate topological errors. However, the new generated small plotsare not existed logically, which need to be merged into the large area around the site. To modifying the graphics, users need to use the editing functions of ArcMap.

\subsubsection{Editing attribute data}

There are many block polygons in a CAD file,and each block polygon has its own figure number.The attribute information of the plot is derived from outside survey,and the generally stored in the excel table together with the corresponding figure number.In ArcGIS,the block and the outer excel table are associated byfigure numberand the graph information and attribute information are saved.

Afterconversing the blockpattern and the figure number in CAD to ArcGIS format, the block pattern will change intoplanar data, the figure numberwill change intopoint data, andnumbering information will be stored in the attribute table of point data. In the use of spatial association, ArcGIS canhelp toassign thepoint data's attribute information ( which is called" figure number") of each map.Then the software can hang the outside excel form to the map according to the figure number,which becomesattribute information of the map. 


\subsubsection{Hierarchically exportdata}

In the process of land intensive use evaluation, the survey of the lands ( has been built, being built or cannot be built) is needed.the survey contains the land nature and used land range .In order to statistic these kinds of land mentioned above more efficiently, users can use ArcGIS to statistic, summary or export data.

When hierarchically exporting data according to different land types,the basis is the field code in theattribute information. Inthe field code,the value with the beginning of "A", such as A2, A4, A51, represents the land has been built, the value with the beginning of "B", such asB21, B22, represents the land being built, the value with the beginning of "C", such asC1, C3 ,represents the land cannot be built. According to its law, the python window set in Arcmap will edit the python script to hierarchically export data automatically.The operating window of python is shown in "Fig.3".

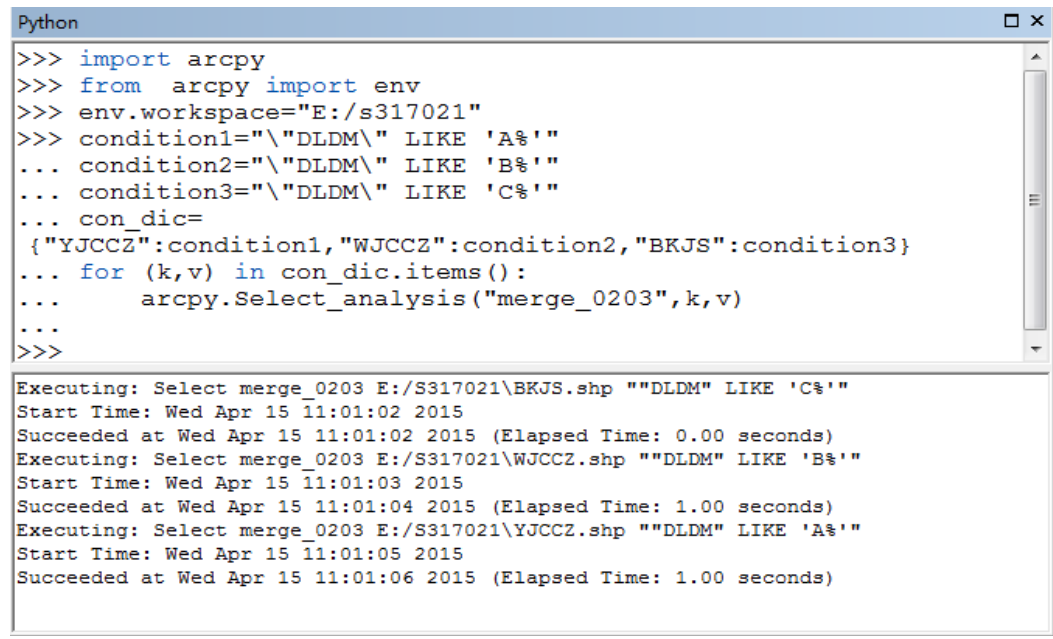

Figure 3. data window of python's hierarchically export Users can export the codes so that they can be reused in other data.

\subsection{Production of thematic maps}

Thesubmitted results map during the land intensive use evaluation work should include land use status map, typical industrial enterprise distribution, land intensive use potential distribution map,management of land intensive use potential distribution map and other thematic maps. By using the cartography function of ArcGIS,users can render layers, insert text in a specified style, scale, compass, legend and other elements according to the requirements.In addition, users can specify the size and layout of the paper. At the end, the resolution and output format shall be pointed. The final result is shown in "Fig.4".

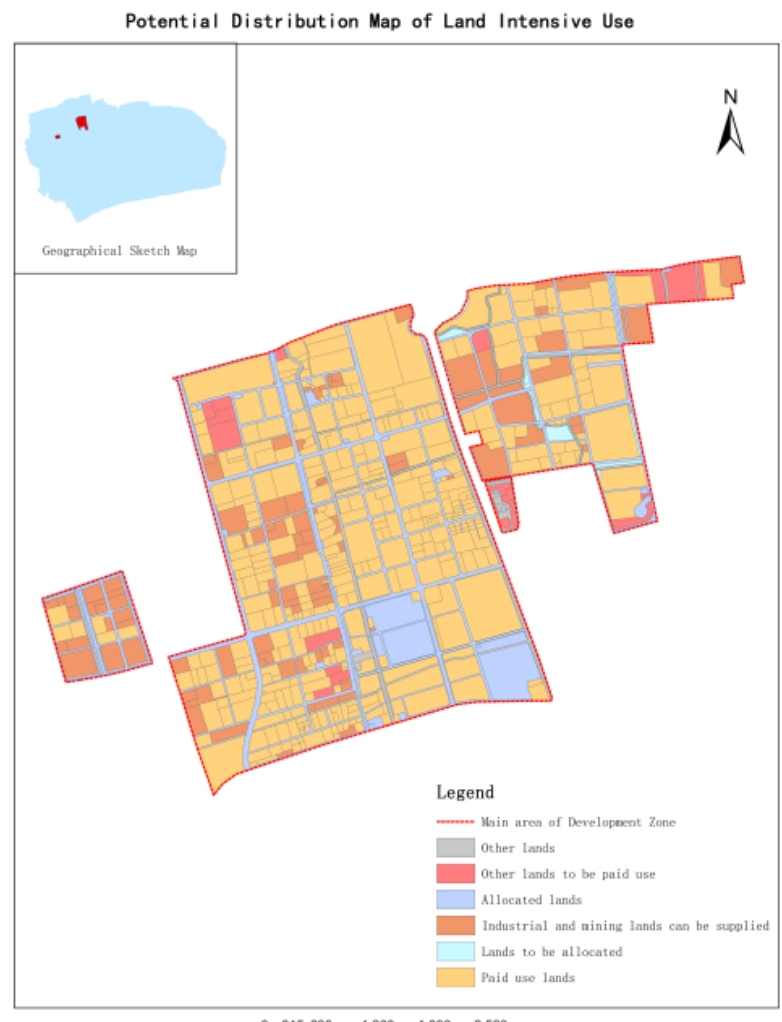

Figure 4. Thethematic map made by Arcmap 


\section{SUMMARY}

On the whole, ArcGISprovides a good technical support for the land intensive use evaluation work,helps to achieve data acquisition, processing and output efficiently. In the future, ArcGIS will play a more important role and help a lot in the process of land intensive use evaluation.

(1) Do kinds of overlap analysis with vector map of the future and the one existed now,which will providereference for the change of land use and provide dynamic evaluation data.

(2)Use the 3D viewfunctionof the ArcGIS to build a more intuitive three-dimensional view based in Building layers,which are calculated according to land volume rate, basal area, construction area and other data.

(3)Combine ArcGIS with othersoftware tools, such as AutoCAD, FME, Google Earth, CorelDraw and Photoshop, to achieve the complementary function and get a better result with less cost.

\section{REFERENCES.}

[1] Song Xiaodong, Niu Xinyi. Geographical information system practice tutorial:ArcGIS9.x[M]. Beijing: Science Press, 2011.

[2] Chi Jian. Proficient in ArcGIS system [M]. Beijing: Tsinghua University press, 2011.

[3] DangQing.Research on urban land price and land intensive use based on GIS[D]. Chengdu:Chengdu University of Technology,2013.

[4] Chen Shupeng.Geographic Information Systems[M] . Beijing: Science Press, 1999.1.

[5] Zhang Hong.Algorithm Based On Geographic Information System[M]. Beijing: Science Press, 2006.6

[6] Zhao Jingliang, Li Zhigang.AutoCAD 2004 and AutoLISP Development Technology[M]. Tsinghua University press,2004.4.

[7] Mu Hehai. Spatial Data Structure and Processing Technology of Geographic Information System (GIS)[M]. Beijing: Surving and Mapping Paress, 1997.

[8] Wu Lixing. Principle and Algorithm of Geographic Information System [M]. Beijing: Science Press,2003.

[9] YangDayuan,ZhouShenglu.Theory and method of urban land evaluation[M].Science Press,2009.9.

[10] Wang Zhanquan, ZhaoSisi, XuHui.GIS Development Projects Case Study[M].POSTS \& TELECOM PRESS 2005.10. 